\title{
Synthesis of Monodisperse InP Quantum Dots: Use of an Acid-Free Indium Carboxylate Precursor
}

\author{
Léo Angelé, ${ }^{[a][b]}$ Sébastien Dreyfuss, ${ }^{[b]}$ Benoit Dubertret, ${ }^{*[b]}$ and Nicolas Mézailles ${ }^{*[a]}$
}

[a] Laboratoire Hétérochimie Fondamentale et Appliquée (LHFA), Université Toulouse 3-Paul Sabatier and CNRS UMR 5069, 31062 Toulouse Cedex 9, France, France

[b] Nexdot, 102 Avenue Gaston Roussel, 93230 Romainville, France

KEYWORDS: Indium phosphide quantum dots, kinetics, precursors, absorption, size focusing, core-shell

\begin{abstract}
The role of indium carboxylate precursors in the synthesis of monodisperse InP quantum dots was investigated. The reaction between acid-free indium palmitate and tris(trimethylsilyl)phosphine $\left(\mathrm{P}(\mathrm{TMS})_{3}\right)$ was monitored using high-temperature ${ }^{31} \mathrm{P}-\mathrm{NMR}$, indicating the presence of a single molecular phosphorus species throughout the duration of the reaction. The addition of varying amounts of carboxylic acid and its effects on both the reaction kinetics and the optical properties of InP QDs was studied. In the presence of acid, rapid protonation of $\mathrm{P}(\mathrm{TMS})_{3}$ led to the formation of a mixture of four $\mathrm{H}_{\mathrm{x}} \mathrm{P}(\mathrm{TMS})_{3-\mathrm{x}}$ species, resulting in the poorer controlled formation of InP nanocrystals. Upon deposition of a gradated $\mathrm{ZnSeS}$
\end{abstract}


shell on the synthesised InP core, luminescent quantum dots were obtained (QY 67\%; PL: FWHM 40nm).

\section{INTRODUCTION}

Quantum dots (QDs) have already reached applications in the lighting and display markets, with the first-generation of commercially viable QDs based primarily on cadmium. ${ }^{1}$ Due to its toxicity, the use of cadmium is drastically limited by international regulations such as the Restriction of Hazardous Substances Directive (RoHS) in Europe. In response to this, academic and industrial researchers have pursued the development of more environmentally friendly materials such as indium phosphide (InP) QDs. ${ }^{2,3}$ So far, the optical properties of InPbased QDs have lagged behind that of Cd-based QDs for several reasons. Cui et al. showed that the broad emission peaks of InP QDs was related to their size polydispersity, since sharp optical properties were obtained with singular QD particles. ${ }^{4}$ More recent work by Janke et al. showed that other factors, such as surface and lattice defects of InP quantum dots, were also contributing to the broadening of the emission linewidth. ${ }^{5}$ Nevertheless, limiting the size distribution of InP QDs remained a critical factor in order to attain these desirable optical properties. Difficulties arising during the synthesis of InP QDs were attributed mainly to the source of phosphorus during the reaction. The pyrophoric, hazardous and readily hydrolysed phosphorus precursor, tris(trimethylsilyl)phosphine $\left(\mathrm{P}(\mathrm{TMS})_{3}\right)$, required the use of an inert atmosphere throughout the entire synthesis with a poor control over the reactivity of the compound. In the presence of an in situ generated indium carboxylate precursor, $\mathrm{P}(\mathrm{TMS})_{3}$ was reported to have rapidly depleted, leading to the growth of InP nanocrystals via a poorly controlled ripening process. ${ }^{6}$ To limit this effect, the groups of Bawendi and Cossairt implemented the use of less reactive silylphosphines 
and germylphosphines such as $\mathrm{P}\left(\mathrm{SiPh}_{3}\right)_{3}$ or $\mathrm{P}\left(\mathrm{GeMe}_{3}\right)_{3}$, leading to slightly better control over polydispersity. ${ }^{7,8}$ Other research groups investigated further into alternative phosphorus sources such as $\mathrm{P}_{4},{ }^{9} \mathrm{PCl}_{3},{ }^{10,11} \mathrm{PH}_{3}{ }^{12,13}$, but with little improvement to the quality of the InP QDs. The most promising recent developments relied on the use of aminophosphines $\mathrm{P}\left(\mathrm{NR}_{2}\right)_{3}$, providing a more accessible route to InP QD synthesis albeit without improving size polydispersity issues. ${ }^{14-}$ 16

The indium precursor remained another equally important parameter for the reaction which has been rarely addressed. Notably, Bawendi et al. showed the importance of controlling the amount of water present in the reaction and recently Peng et al. demonstrated improvements on optical properties by additional phosphine ligands coordinating the indium centre before the start of the reaction. ${ }^{17,18}$

However, in most InP QD syntheses an indium carboxylate precursor bearing long aliphatic chains is generated in situ from In(acetate $)_{3} \cdot{ }^{19}$ Narayanaswamy et al. showed that under the conditions used, only about two out of three acetate ligands are displaced from the indium centre, implying that approximately one equivalent of the non-volatile long chain carboxylic acid remained in the reaction medium throughout the entire process..$^{20}$ According to literature, this free fatty acid would have an effect on the synthesis of InP QDs. Firstly, as demonstrated by Cossairt and co-workers, the free fatty acid would rapidly hydrolyse the phosphorus precursor $\mathrm{P}(\mathrm{TMS})_{3}$ to form mixtures of $\mathrm{H}_{\mathrm{x}} \mathrm{P}(\mathrm{TMS})_{3-\mathrm{x}}(\mathrm{x}=1$ to 3$)$ with different reactivities towards the $\mathrm{In}$ precursor. ${ }^{21}$ Secondly, Chaudret's group showed that free fatty acids, upon heating at InP QD synthesis temperatures $\left(250^{\circ} \mathrm{C}\right.$ or more), would undergo ketonization thereby releasing water and ketone molecules in the process. In turn, these water molecules would lead to surface oxidation of the InP QDs. ${ }^{22,23}$ In other words, working under air and water-free conditions would not be 
sufficient for good reaction control if free acid was present in the medium either at the beginning of the reaction or generated in situ. The working hypothesis for this study consisted of the principle that an $\operatorname{In}(\mathrm{RCOO})_{3}$ precursor, free of acid, would lead to an improved control over InP QD formation during synthesis.

In the present work, a simple synthesis of such acid free In(palmitate) $)_{3}$ complex was carried out. InP QDs were synthesized from pure Indium complex with controlled amounts of additional carboxylic acid and compared. The absence of acid was shown to provide a slow growth of QDs without ripening occurring, believed to be linked to the presence of a single molecular P species. In the presence of increasing amounts of acid, faster, less controlled reactions occurred, linked to the presence of four reactive molecular $\mathrm{P}$ derivatives. This precise understanding ensured InP QDs were obtained within a controlled, size-focusing regime featuring excellent optical properties after deposition of a protective shell via a simple heat up process. To the best of our knowledge, greater optical properties were only obtained via hotinjection methods or through the use of zinc carboxylates as additives. ${ }^{23}$ However, using zinc carboxylates results in irregular and detrimental core doping, whilst hot-injection syntheses are significantly harder to scale up. ${ }^{5}$

\section{RESULTS AND DISCUSSION}

As mentioned above, in most InP QDs syntheses the indium carboxylate precursor is generated in situ. ${ }^{19}$ This first step is performed by reacting indium (III) acetate, a commercial precursor, with three equivalents of myristic, palmitic or oleic acid, at $c a 120^{\circ} \mathrm{C}$ for a few hours under dynamic vacuum. This dynamic vacuum speeds up the reaction process by eliminating acetic acid generated as a by-product of the process. ${ }^{25,26}$ However, under these conditions, the 
reaction was unable to run to completion and a mixture of $\operatorname{In}(\mathrm{RCOO})_{3-\mathrm{x}}\left(\mathrm{CH}_{3} \mathrm{COO}\right)_{\mathrm{x}}$ with $\mathrm{x} c a 1$ is formed. ${ }^{20}$ To carry out the present study, we set out to isolate pure indium palmitate complex $\operatorname{In}(\text { Palm })_{3}$. In order to ensure complete substitution of all three acetate ligands, an excess of palmitic acid (10 equiv., solvent of the reaction) was used as well as longer reaction times (4 hours at $110^{\circ} \mathrm{C}$ under dynamic vacuum) (figure 1). After the reaction, the crude product was thoroughly washed as described in ESI, in order to remove the excess of palmitic acid and any trace remnants of acetic acid. ${ }^{25}$ Finally, the product was dried under vacuum at $100^{\circ} \mathrm{C}$ for 6 hours.

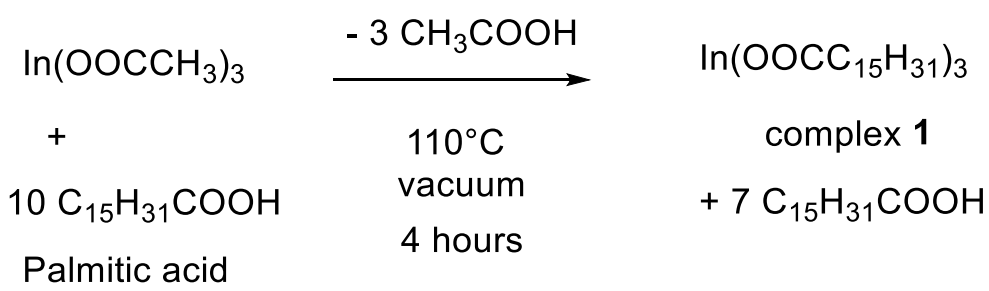

Figure 1. Optimized synthesis of $\operatorname{In}(\mathrm{Palm})_{3}$; where palmitic acid is both the solvent and the reactant.

The purity of our In(Palm $)_{3}$ sample was demonstrated by NMR and IR spectroscopy. The ${ }^{1} \mathrm{H}$ NMR spectrum of the isolated complex did not show a singlet corresponding to the $\mathrm{CH}_{3}$ of the acetate moiety, expected at around $1.8 \mathrm{ppm}$ which suggested the formation of $\operatorname{In}(\mathrm{Palm})_{3}$.(ESI, part 1) Note that while our work was in progress, Tamang et al. reported the synthesis of $\operatorname{In}(\mathrm{RCOO})_{3}$ from the reaction of $\mathrm{InCl}_{3}$ with 3 equiv. of carboxylic acid in the presence of an organic base. This work is dedicated to the development of a general synthesis of a wide variety of metal carboxylate complexes. ${ }^{27}$ In the IR spectrum, no characteristic signal for the $\mathrm{COOH}$ moiety could be found (figure 2). Superposition of spectra for palmitic acid and $\operatorname{In}(\mathrm{Palm})_{3}$ complex 1 showed the absence of the $\mathrm{O}=\mathrm{C}$ stretch band from the acid at $1700 \mathrm{~cm}^{-1}$ and the presence of two O-C stretch bands at 1580 and $1540 \mathrm{~cm}^{-1}$ of $\operatorname{In}(\mathrm{Palm})_{3}$. 


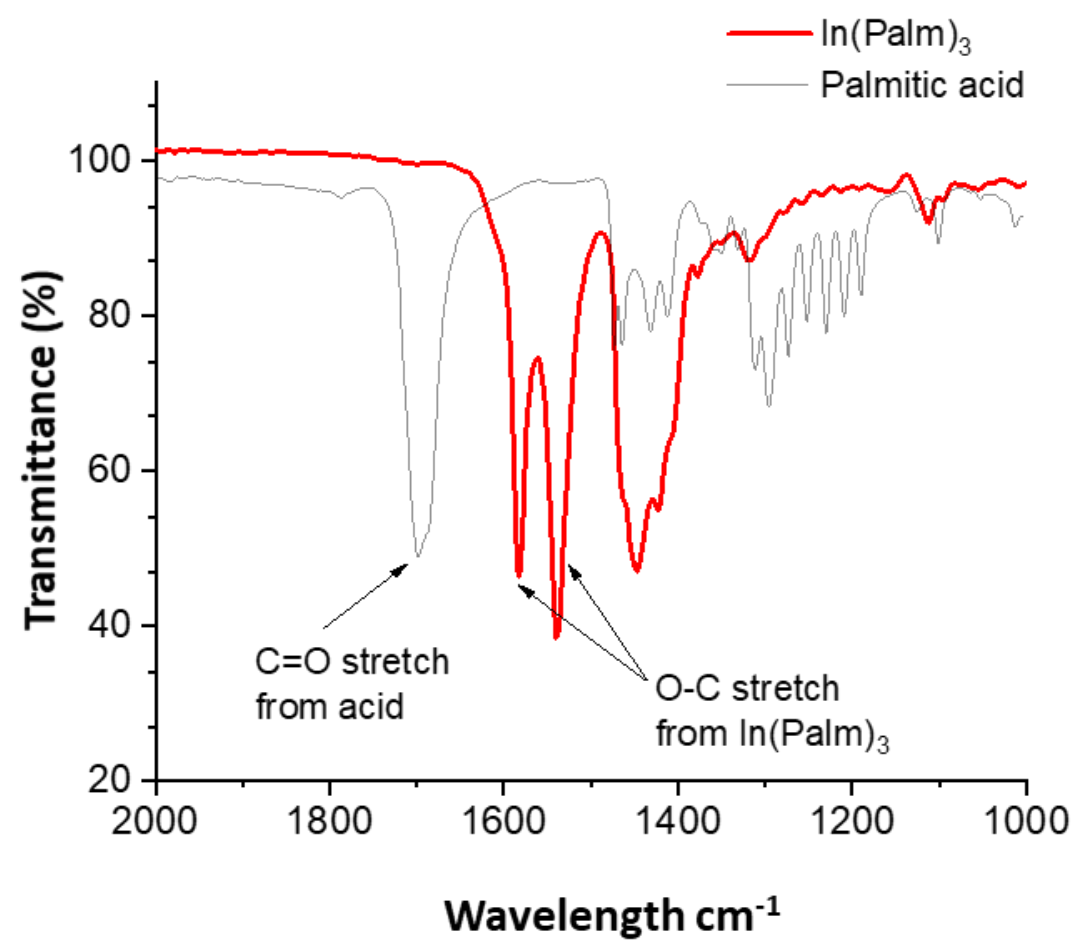

Figure 2. Infra-red spectra of $\operatorname{In}(\mathrm{Palm})_{3}$ complex 1 and palmitic acid (2000-1000 $\mathrm{cm}-1$ region). An additional, indirect, proof of purity of complex 1 was performed upon addition of minute amounts of palmitic acid (5\% by mass), whereby the signal corresponding to $\mathrm{COOH}$ would appear once more at $1700 \mathrm{~cm}^{-1}$ (figure S4). ${ }^{28}$ The complex 1 was subsequently stored in an Ar-filled glovebox in order to prevent possible $\mathrm{H}_{2} \mathrm{O}$ coordination at the Lewis acid Indium centre. With this acid and water-free In precursor, the reaction with $\mathrm{P}(\mathrm{TMS})_{3}$ in a 1:1 ratio in 1Octadecene (ODE) at $80^{\circ} \mathrm{C}$ was monitored by ${ }^{31} \mathrm{P}$ NMR spectroscopy (in the presence of $\mathrm{PPh}_{3}$ as internal standard). The reagents and solvent were mixed in a J-Young NMR tube at room temperature inside an Ar-filled glovebox (see ESI for details). The NMR tube was then inserted into the probe of the NMR spectrometer and heated to $80^{\circ} \mathrm{C}$ while ${ }^{31} \mathrm{P}$ NMR spectra were recorded every 263s to allow a quantitative analysis of the spectra (see figure S7 and S8 for full spectra at $\mathrm{t}=0$ and $\mathrm{t}=22 \mathrm{~min} 15 \mathrm{~s})$. As shown on figure 3 , which corresponds to zoomed parts of 
the full spectra where $\mathrm{P}(\mathrm{TMS})_{3-\mathrm{x}} \mathrm{H}_{\mathrm{x}}$ can be observed, only a single signal corresponding to $\mathrm{P}(\mathrm{TMS})_{3}$ was observed throughout the reaction.

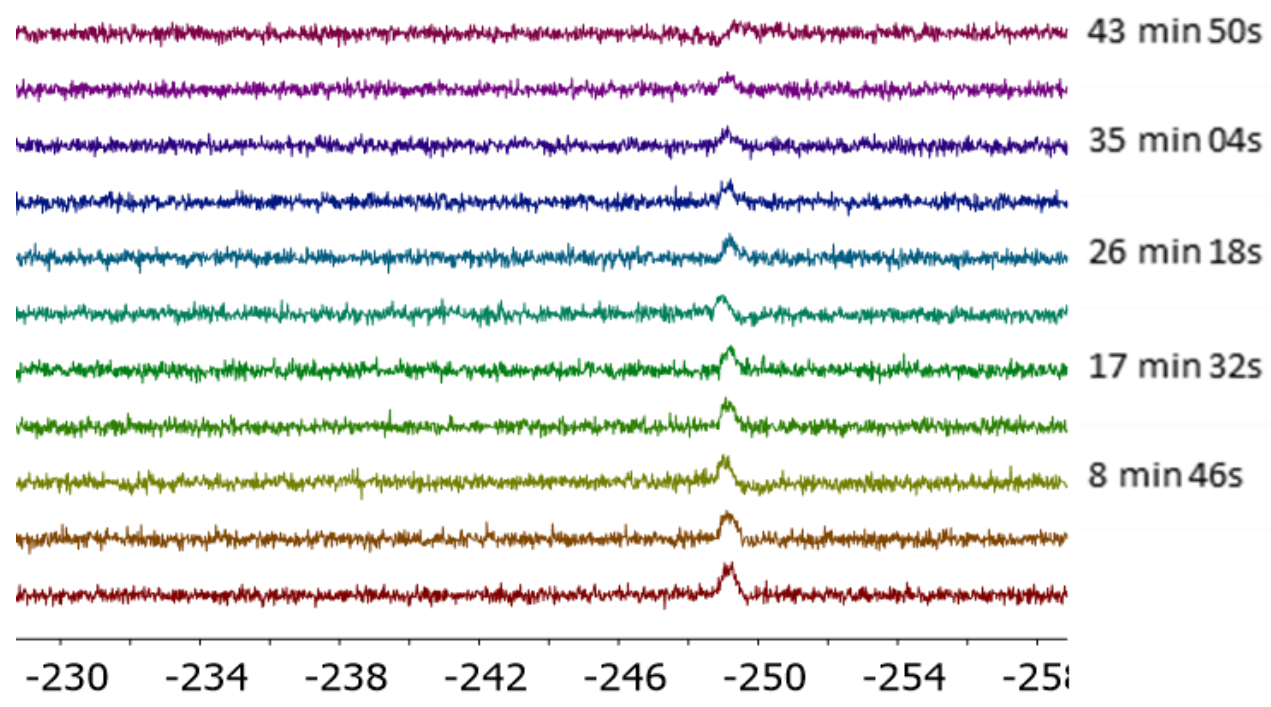

Figure 3. Monitoring of reaction between $\operatorname{In}(\mathrm{Palm})_{3}$ and $\mathrm{P}(\mathrm{TMS})_{3}$ at $80^{\circ} \mathrm{C}$, zoom of the spectra collected every $263 \mathrm{~s}$. The only signal corresponds to $\mathrm{P}(\mathrm{TMS})_{3}$

Note that the apparent peak broadness of $\mathrm{P}(\mathrm{TMS})_{3}$ can be explained by the poor shimming process in NMR resulting from presence of solid material $\left(\operatorname{In}(\mathrm{Palm})_{3}\right.$ is poorly soluble in D8-tol needed for the high temperature experiment) inside the NMR tube through most of the reaction $^{29}$ The intensity of the $\mathrm{P}(\mathrm{TMS})_{3}$ resonance decreased over time and stabilized after $1 \mathrm{hr}$ and 40minutes of reaction to a remaining $15 \%$ of the initial amount ( $v s$ triphenylphosphine (TPP), used as an internal standard). This first experiment demonstrated that in the absence of an external source of acid only one molecular phosphorus species, $\mathrm{P}(\mathrm{TMS})_{3}$, was present during the synthesis of InP, with figure 4 showing the proposed mechanism for that reaction. It also 
highlighted that a temperature higher than $80^{\circ} \mathrm{C}$ was needed in order to fully convert all the phosphorus precursor.

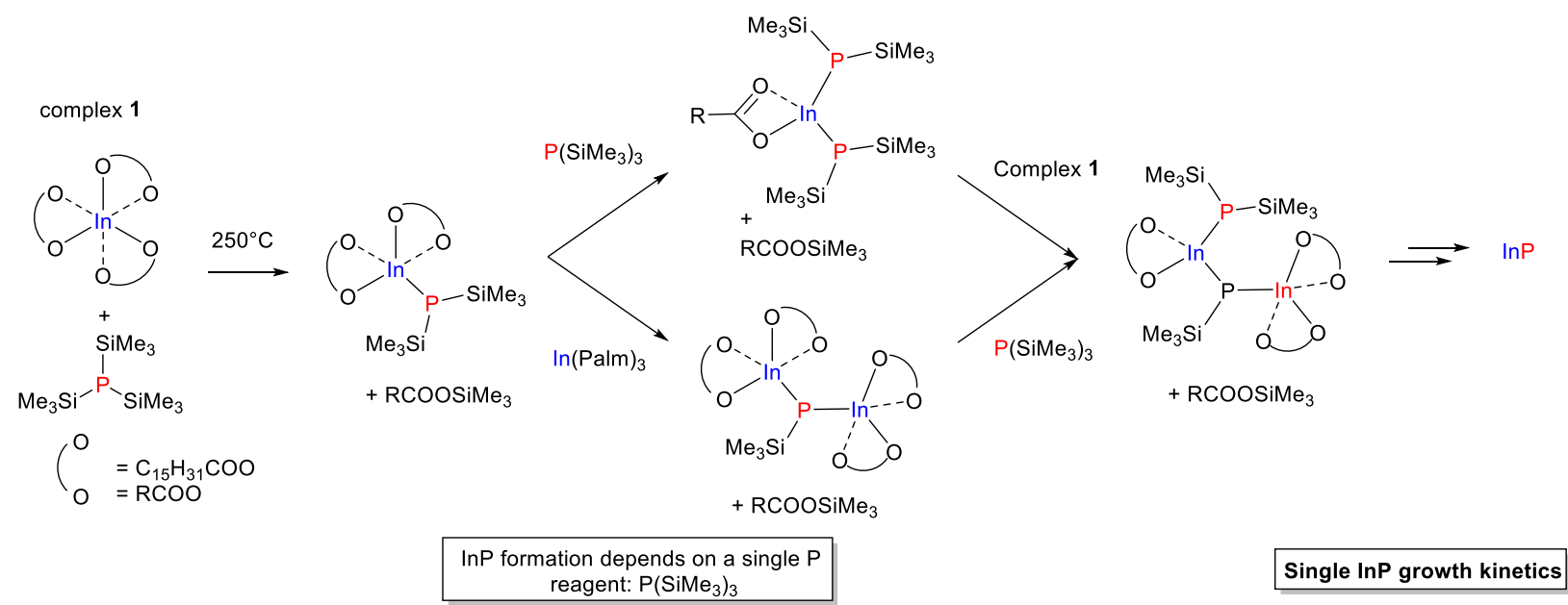

Figure 4. Proposed mechanism for the initial steps of the InP synthesis with likely intermediates, highlighting the formation of $\mathrm{RCOOSiMe}_{3}$ as the sole by product of the stepwise In-P bond formations.

A control experiment was carried out under the same conditions without the $\operatorname{In}(\mathrm{Palm})_{3}$ species. ${ }^{31} \mathrm{P}$ NMR showed no evolution of the $\mathrm{P}(\mathrm{TMS}){ }_{3}$ peak, which implied that the phosphorus precursor reacted solely with the indium carboxylate complex. This suggested that the final stages of crystalline InP QD formation require higher thermal activation. In parallel, an identical reaction was followed by UV-visible spectroscopy. Although the solution took a bright red colour within 15 minutes, no defined absorption peak could be observed (figure S6). This confirms that $80^{\circ} \mathrm{C}$ was neither enough to obtain crystalline quantum dots, nor to have formed magic-size clusters (MSC). ${ }^{26}$ The effect of temperature on the reaction was therefore studied using an approach inspired from Lee et $a .^{25}$. This involved heating a mixture of $\operatorname{In}(\mathrm{Palm})_{3}(1.0$ equiv.), $\mathrm{P}(\mathrm{TMS})_{3}\left(0.8\right.$ equiv.) and trioctylphosphine (TOP) in ODE under $\mathrm{N}_{2}$. Note that the ratio $\mathrm{P} / \mathrm{In}$ of 0.8 was selected based on literature syntheses. ${ }^{30}$ With higher $\mathrm{P} / \mathrm{In}$ ratios, rapid 
aggregation of a dark product was observed rather than well dispersed InP QDs. The mixture was heated to $305^{\circ} \mathrm{C}$ in $20 \mathrm{~min}$ and kept at this temperature for a further 10 minutes. The reaction was monitored using UV-visible spectroscopy by taking aliquots at various temperatures, which are presented in figure $5 \mathrm{a}$.
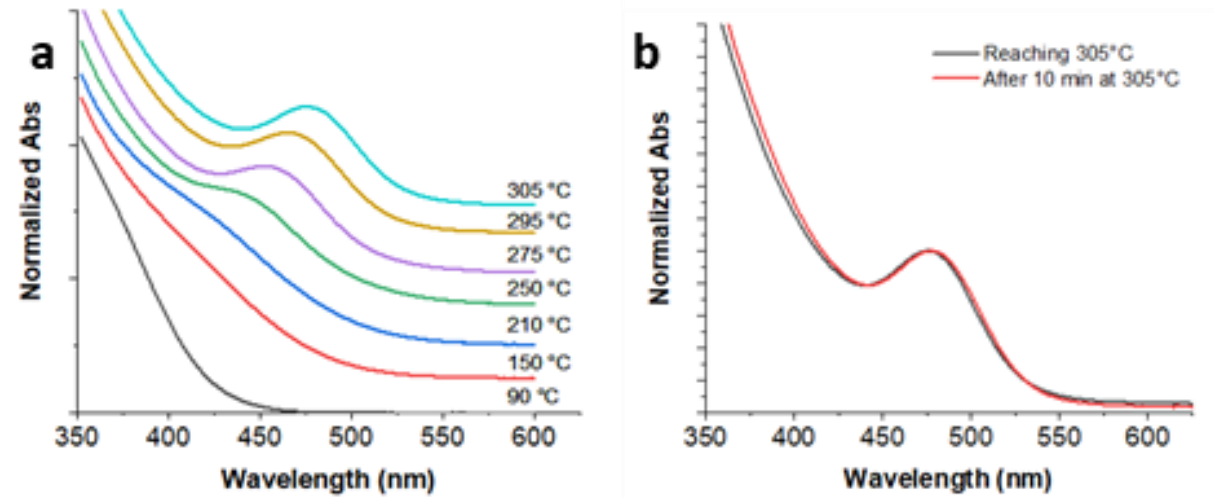

Figure 5. (a) Absorption spectra of the $\mathrm{InP}$ cores at various temperature without acid (b) Absorption spectrum when reaching $305^{\circ} \mathrm{C}$ and after $10 \mathrm{~min}$ at $305^{\circ} \mathrm{C}$

At temperatures between $90^{\circ} \mathrm{C}$ and $150^{\circ} \mathrm{C}$, the reaction mixture remained colourless, confirmed by weak absorption in the visible light range. Between $150^{\circ} \mathrm{C}$ and $200^{\circ} \mathrm{C}$, a light colouration was observed, yet no excitonic features distinctive of quantum dots were visible. A broad excitonic peak around $440 \mathrm{~nm}$ corresponding to $\mathrm{QD}$ formation appeared only at $250^{\circ} \mathrm{C}$. When the temperature was increased further, the absorption peak shifted to higher wavelengths and sharpened. Notably, this sharpening of the absorption peak indicated that the growth occurred during a size focusing regime. Once the temperature reached $305^{\circ} \mathrm{C}$, the $\mathrm{UV}$-vis spectrum of the reaction mixture stopped evolving, indicating the depletion of the phosphorus precursor. No ripening or degradation processes were observed, even after further heating at $305^{\circ} \mathrm{C}$ for 10 minutes, as shown in figure $5 \mathrm{~b}$. These results strongly differ from those of previous studies on InP QDs synthesized with in situ generated Indium carboxylates and $\mathrm{P}(\mathrm{TMS})_{3}{ }^{2}$ Indeed, it has 
been reported that the main difficulty for controlling the synthesis of InP QDs was due to a rapid and uncontrollable consumption of $\mathrm{P}(\mathrm{TMS})_{3}$, and that this growth occurred solely by ripening processes. Therefore, it was considered impossible to reach a size focusing regime without using multi-injection techniques or additives such as zinc carboxylates. ${ }^{30,31}$ This work showed that these detrimental processes can be circumvented using an indium precursor free of carboxylic acid.

To fully understand the role of the carboxylic acid, similar experiments were carried out with controlled, precise amounts of added oleic acid. Oleic acid was chosen over palmitic acid as its liquid state at room temperature simplified the weighing out of minute amounts, around $1 \mathrm{mg}$, with higher accuracy. For direct comparison, the reactions were also conducted in J-Young NMR tubes and monitored at $80^{\circ} \mathrm{C}$ by NMR spectroscopy. Note that each reaction was carried out at least twice, with identical results. As reported in the literature, $\mathrm{P}(\mathrm{TMS})_{3}$ was readily hydrolysed in a fast and non-selective process in the absence of an In precursor. ${ }^{20}$ With sub-stoichiometric amounts of acid, a mixture of four compounds $\mathrm{HP}(\mathrm{TMS})_{2}, \mathrm{H}_{2} \mathrm{P}(\mathrm{TMS})$ and $\mathrm{PH}_{3}$ together with $\mathrm{P}(\mathrm{TMS})_{3}$ was observed. The relative proportions of these compounds depended on the initial amount of acid and these ratios did not change in solution, even when heated. Reactions between $\operatorname{In}(\text { palm })_{3}: \mathrm{P}(\mathrm{TMS})_{3}:$ oleic acid with ratios $1.0: 0.8: \mathrm{x}$, with $\mathrm{x}=0.1$ to 1.0 were followed by ${ }^{31} \mathrm{P}$ NMR spectroscopy. As a representative example, the spectra collected from the experiment with $\mathrm{x}=1.0$ are presented in Figure 6. 


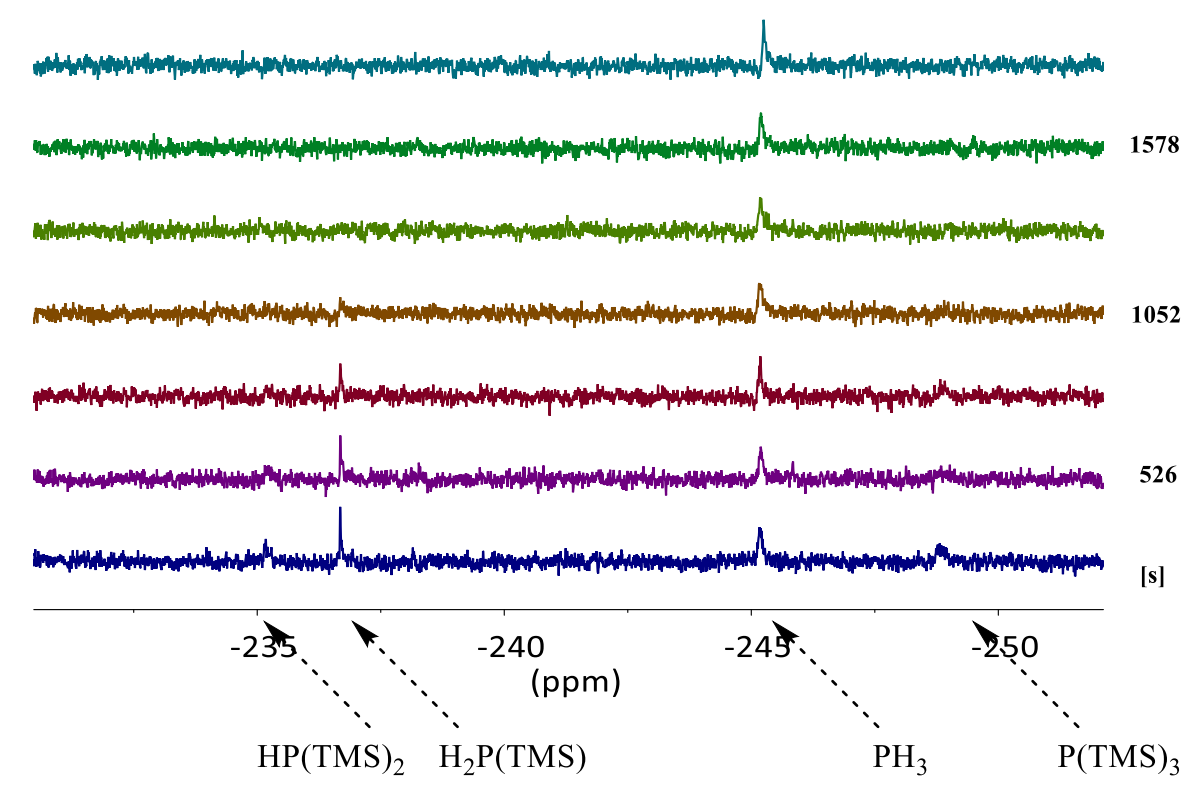

Figure 6. Monitoring of reaction between $\operatorname{In}(\mathrm{Palm})_{3}: \mathrm{P}(\mathrm{TMS})_{3}$ :oleic acid in 1.0:0.8:1.0 ratio at $80^{\circ} \mathrm{C}:{ }^{31} \mathrm{P}\left\{{ }^{1} \mathrm{H}\right\}$ NMR spectra recorded each $263 \mathrm{~s}$.

The experiments carried out with different ratios are presented in the ESI (figures S9 to S13). Integration of all four signals throughout the reaction was used to gauge the evolution of precursor concentrations. The spectra highlight that each of the phosphorus derivatives possessed a different reactivity towards the indium complexes formed during the reaction. Specifically, it appeared that the $\mathrm{HP}(\mathrm{TMS})_{2}$ species decreased faster than the $\mathrm{P}(\mathrm{TMS})_{3}$ and $\mathrm{H}_{2} \mathrm{PTMS}$, themselves faster than $\mathrm{PH}_{3}$ in accord with previously reported literature. ${ }^{7}$ Importantly, the monitoring showed no further evolution of the total amount of $\mathrm{P}$ derivatives after 15 minutes, with only the $\mathrm{PH}_{3}$ remaining. The time needed to reach this plateau depended on the amount of free carboxylic acid, ranging from 15 minutes when 1.0 equivalent of acid was present, to 100 minutes without acid (ESI figures S5 and S9 to S12). This demonstrated the difference in reactivity of the four phosphorus derivatives. This led to reasonably postulate that such differences in reactivity already seen at $80^{\circ} \mathrm{C}$ would have an impact on the growth control of InP QDs at higher 
temperatures. A UV-visible spectrum of the mixture resulting from the reaction of $\operatorname{In}(\operatorname{Palm})_{3}$, $\mathrm{P}(\mathrm{TMS})_{3}$, oleic acid in a 1.0:0.8:1.0 ratio at $80^{\circ} \mathrm{C}$ for $2 \mathrm{~h}$ was recorded (figure $\mathrm{S} 13$ ) which did not show any excitonic features corresponding to QDs. Higher temperatures were clearly needed to achieve QDs formation as a result. Considering these results, the effect of the amount of added oleic acid $(0.1,0.3,0.5$, and 1.0 equivalents $)$ on the outcome of the standard QD synthesis at high temperatures was studied. The reactions were monitored by UV-visible spectroscopy of aliquots taken at various temperatures.

A broad absorption feature could already be detected when the temperature reached $90^{\circ} \mathrm{C}$ when only more than 0.3 equiv. acid was used (figure $7 \mathrm{a}$ ). In comparison, without the presence of acid, excitonic features only appeared at temperatures of $250^{\circ} \mathrm{C}$ (figure 5a.). Surprisingly at $90^{\circ} \mathrm{C}$, the reaction with 1.0 equiv. acid seemed to result in smaller-size QDs than the reaction with 0.5 equiv. acid. It was hypothesised that this might have been due to the production of MSCs, as these conditions were similar to the ones used by Cossairt et al. to produce MSCs absorbing at $386 \mathrm{~nm} .^{32}$ To test this hypothesis, further experiments were performed at $80^{\circ} \mathrm{C}$. Without acid, no MSCs were observed while with 1.0 eq of oleic acid, a sharp excitonic feature centred at $365 \mathrm{~nm}$ corresponding to MSCs was observed (Figure S15). These experiments confirmed that with an initial amount of 1.0 equiv. of free acid, MSCs were likely intermediates for the formation of InP QDs, which is consistent with previous literature reports. $^{21}$ At $210^{\circ} \mathrm{C}$, the differences between reactions with various acid amounts were particularly significant as seen in figure $7 \mathrm{~b}$. 

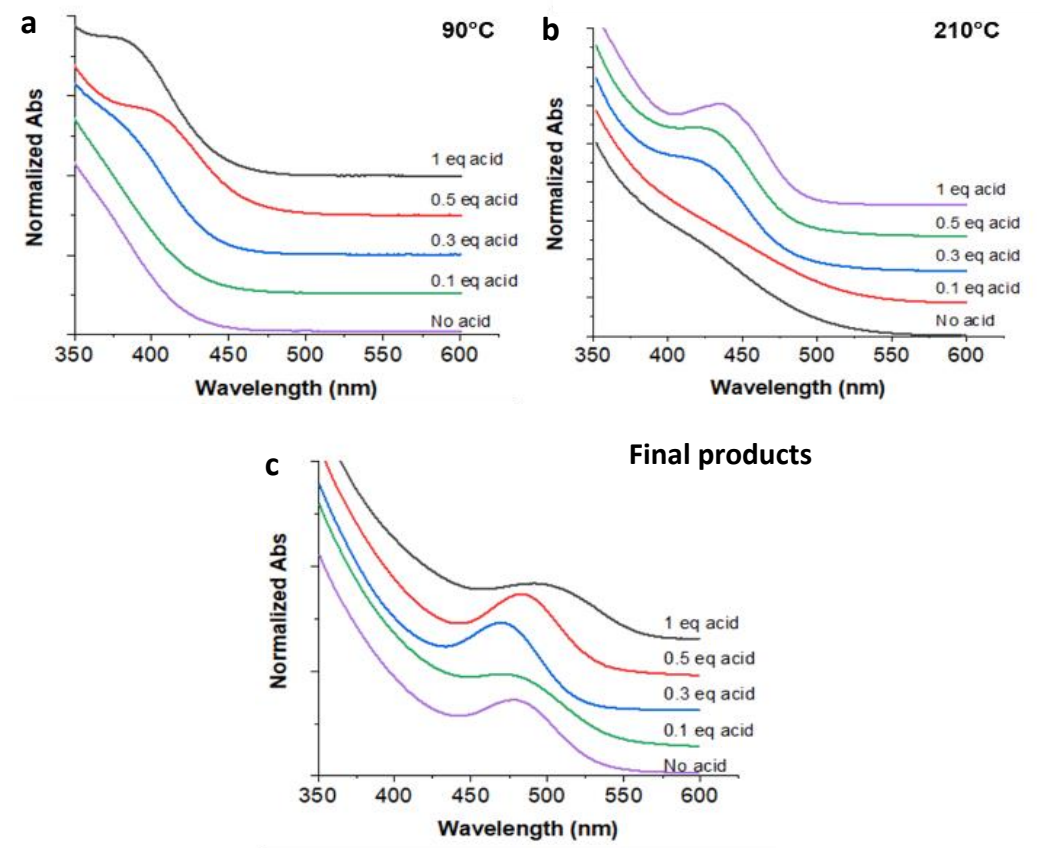

Figure 7. Absorption spectra of reactions at $90^{\circ} \mathrm{C}$ (a), $210^{\circ} \mathrm{C}$ (b) and final products ((c), 10 minutes at $305^{\circ} \mathrm{C}$ ) with various amounts of added acid.

Without acid or with 0.1 equiv., nucleation had barely started and no excitonic feature was present. On the other hand, reactions with 0.3-1.0 equiv. acid displayed a far more defined absorption peak showing QD formation, whereby the signal was even sharper for 1.0 equiv. of acid. Undoubtedly, free acid accelerated the formation of QDs and at this point appeared to be a strongly inducing parameter. The QDs in figure $7 \mathrm{c}$ obtained after 10 minutes at $305^{\circ} \mathrm{C}$, presented very different absorption spectra.

To understand this, the full reaction monitoring for each acid amount was studied in greater detail (figure S16 to S21). The profile of the reaction with one equiv. of acid (figure 8a) was particularly significant. At $90^{\circ} \mathrm{C}$, a broad excitonic feature around $390 \mathrm{~nm}$ was already present, which as mentioned above, could have been attributed to the growth of the $365 \mathrm{~nm}$ absorbing MSCs. Between $90^{\circ} \mathrm{C}$ and $250^{\circ} \mathrm{C}$, a shift of the absorption peak was observed, consistent with InP QD growth. At $250^{\circ} \mathrm{C}$, optimal size dispersity was obtained, as seen by the 
sharpness of the excitonic feature, with a half width at half maximum (HWHM) of $34 \mathrm{~nm}$, taken from the higher wavenumber side of the peak and a peak/valley ratio of 1.29. Upon further heating, InP QDs continued to grow with a shift in the absorption peak from $470 \mathrm{~nm}$ to $500 \mathrm{~nm}$. Although, at the same time the peak broadened, implying an increase in size dispersity due to a detrimental ripening process.
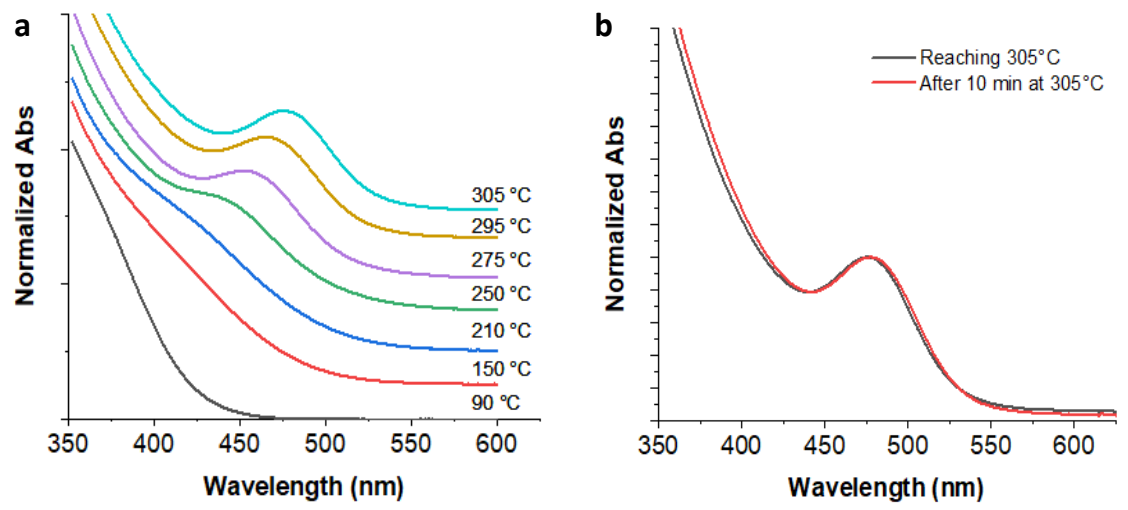

Figure 8. (a) Absorption spectra of the InP cores at various temperature with 1 equiv. acid (b) Absorption spectrum when reaching $305^{\circ} \mathrm{C}$ and after $10 \mathrm{~min}$ at $305^{\circ} \mathrm{C}$.

Importantly, this ripening process continued when the mixture was held at $305^{\circ} \mathrm{C}$ for an additional 10 minutes (figure 8b) leading to a HWHM increase from $42 \mathrm{~nm}$ to $47 \mathrm{~nm}$, and a peak/valley ratio decrease from 1.23 to 1.11 . The reactions with 0.3 and 0.5 equiv. acid (figures S18 and S19 respectively) presented a similar behaviour. They reached an optimal size dispersity at $275^{\circ} \mathrm{C}$ and $295^{\circ} \mathrm{C}$ respectively before a ripening process took place. Conversely, in the absence of added acid, the reaction profile showed a broad excitonic feature at $250^{\circ} \mathrm{C}$ that sharpened with an increase in temperature, showing the growth of QDs with size focusing and without ripening after 10 minutes at $305^{\circ} \mathrm{C}$ (figures $5 \mathrm{a}$ and b). Most importantly, the absence of ripening allowed for greater control over the reaction as no quenching was required. The reaction with 0.1 equiv. of acid differed from the others with the absence of a well-defined peak between 
$210^{\circ} \mathrm{C}$ and $305^{\circ} \mathrm{C}$ (figure S17). This result was consistent with a recent report by Peng et al. who obtained a similar reaction profile for a reaction through a heat-up setup while working with $10 \%$ free acid in the medium. ${ }^{17}$ This could be due to the presence of two nucleation regimes: a first one starting at temperatures below $100^{\circ} \mathrm{C}$ due to the free acid, and a second one occurring at temperatures higher than $200^{\circ} \mathrm{C}$ comparable to the conditions without free acid. This could imply a prolonged nucleation, leading to greater size polydispersity, consistent with results by Cossairt et al. who demonstrated that longer nucleation times for InP quantum dots during synthesis was detrimental to reaching a homogenous size dispersity. ${ }^{8}$ Thus, even trace amounts of free acid does lead to significant size dispersity during the heat-up process, counteracting the prior isolation of the $\mathrm{In}(\mathrm{Palm})_{3}$ precursor.

These findings on the influence of the amount of acid present during the synthesis of InP QDs could be rationalized by the initial steps proposed in figure 9. The acid partly hydrolyses $\mathrm{P}(\mathrm{TMS})_{3}$ to four different compounds $\mathrm{P}(\mathrm{TMS})_{3-\mathrm{x}} \mathrm{H}_{\mathrm{x}}($ with $0<\mathrm{x}<3$ ) which react at different rates with the $\operatorname{In}(\mathrm{Palm})_{3}$ precursor, to form three possible indium complexes, featuring the first In-P bond. For each P-H bond reaction, one equivalent of palmitic acid would be formed, and this acid could in turn react with the mixture of phosphines to once again modify their relative proportion. The acid could equally react with the $(\mathrm{RCOO})_{2} \operatorname{InP}\left(\mathrm{SiMe}_{3}\right)_{2-\mathrm{x}}(\mathrm{H})_{\mathrm{x}}(\mathrm{x}=0.1)$ as well. Further reaction of the three indium complexes would occur with different kinetics due to the significant differences in steric environments at the phosphorus centre. Therefore, this would explain the poor conditions for controlling growth when fatty acid would be present in the medium. Importantly, the proposed mechanism does show that the overall quantity of acid would not change throughout the reaction, which would also be consistent with the ripening process that does occur only in the presence of acid. 


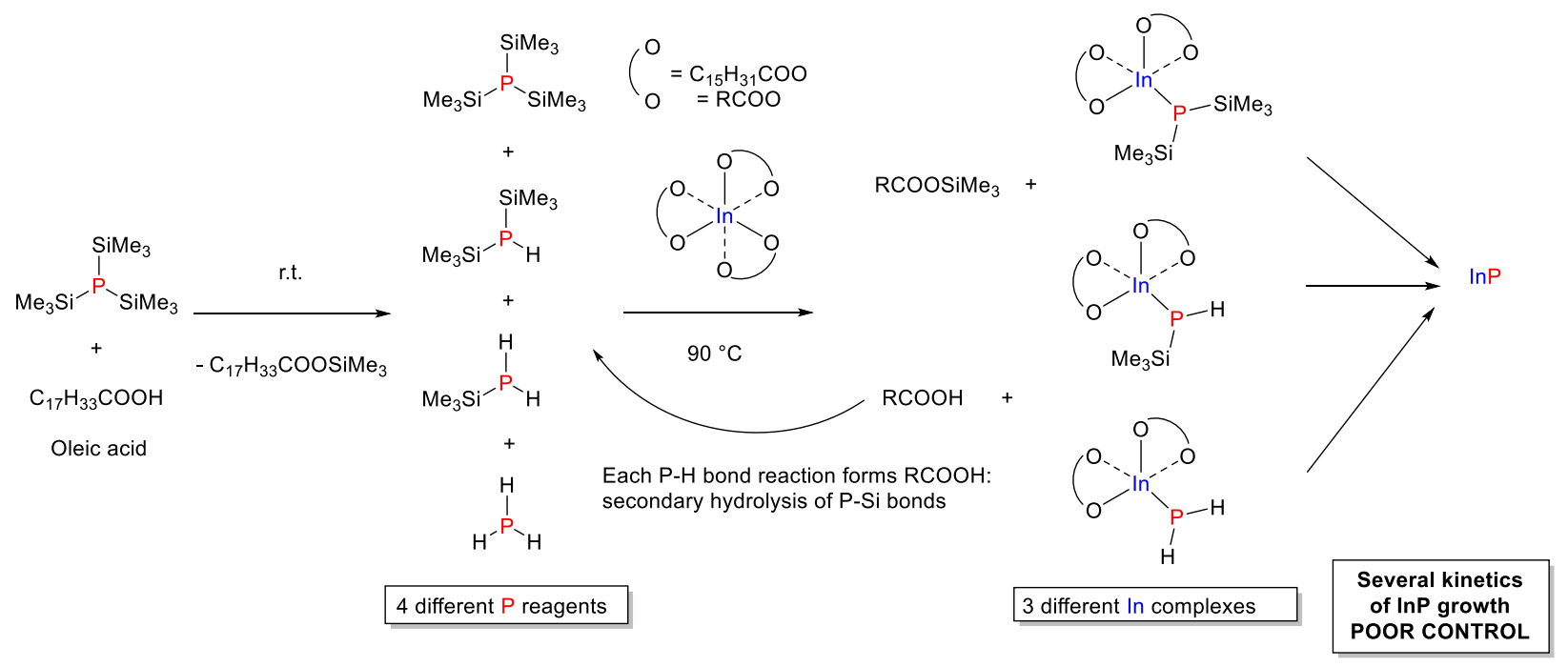

Figure 9. Proposed mechanism of the initial steps of the InP synthesis with likely intermediates, highlighting the formation of $\mathrm{RCOOH}$ as an extra by-product of the stepwise In-P bond formations

A final experiment was carried out to compare these results to a classic procedure from literature using an in situ generated indium precursor. ${ }^{19}$ Anhydrous indium(III) acetate and three equivalents of palmitic acid were mixed at $120^{\circ} \mathrm{C}$ in $\mathrm{ODE}$ for $2 \mathrm{~h}$ under vacuum $(0.3 \mathrm{mbar})$. $\mathrm{P}(\mathrm{TMS})_{3}$ and TOP were added to this precursor solution, then the mixture was heated up to $305^{\circ} \mathrm{C}$ under conditions identical to above. As shown in figure S4.6, the UV-visible monitoring showed an evolution nearly identical to the one when 1.0 equiv. of acid was added to the pure $\operatorname{In}(\text { Palm })_{3}$ complex. Thus, it is believed that some reproducibility issues with the InP QD syntheses were due to the way the indium precursor was generated (time under vacuum, temperature, pressure, amount of initial acid), and therefore on the amount of remaining free fatty acid within the mixture. Having obtained InP QDs from different syntheses we proceeded to evaluate their emissive properties after deposition of a $\mathrm{ZnSeS}$ gradient shell which displayed good luminescent properties due to reduced lattice mismatching. For the deposition of the $\mathrm{ZnSeS}$ shell, zinc stearate was introduced into a crude mixture of the InP cores at room temperature 
under stringent air-free conditions. Then, the solution was heated to $310{ }^{\circ} \mathrm{C}$ with successive injections of TOP-Se and TOP-S in order to form a gradient $\mathrm{ZnSeS}$ shell. Emission spectra were recorded and shown below in figure 10, and PL data presented in table 1. For the acid-free synthesis, the InP/ZnSeS core-shell quantum dot showed an emission wavelength of $550 \mathrm{~nm}$ with a full width at half maximum (FWHM) of $40 \mathrm{~nm}$, and a photoluminescence quantum yield (QY) of $67 \%$. An identical $\mathrm{ZnSeS}$ shell deposition procedure was carried out on an InP cores synthesized with varied amounts of acid. All the final core/shell quantum dots displayed a quantum yield between 60 and $67 \%$.

Table 1. Optical properties of InP/ZnSeS core shell QD depending on the amount of free acid

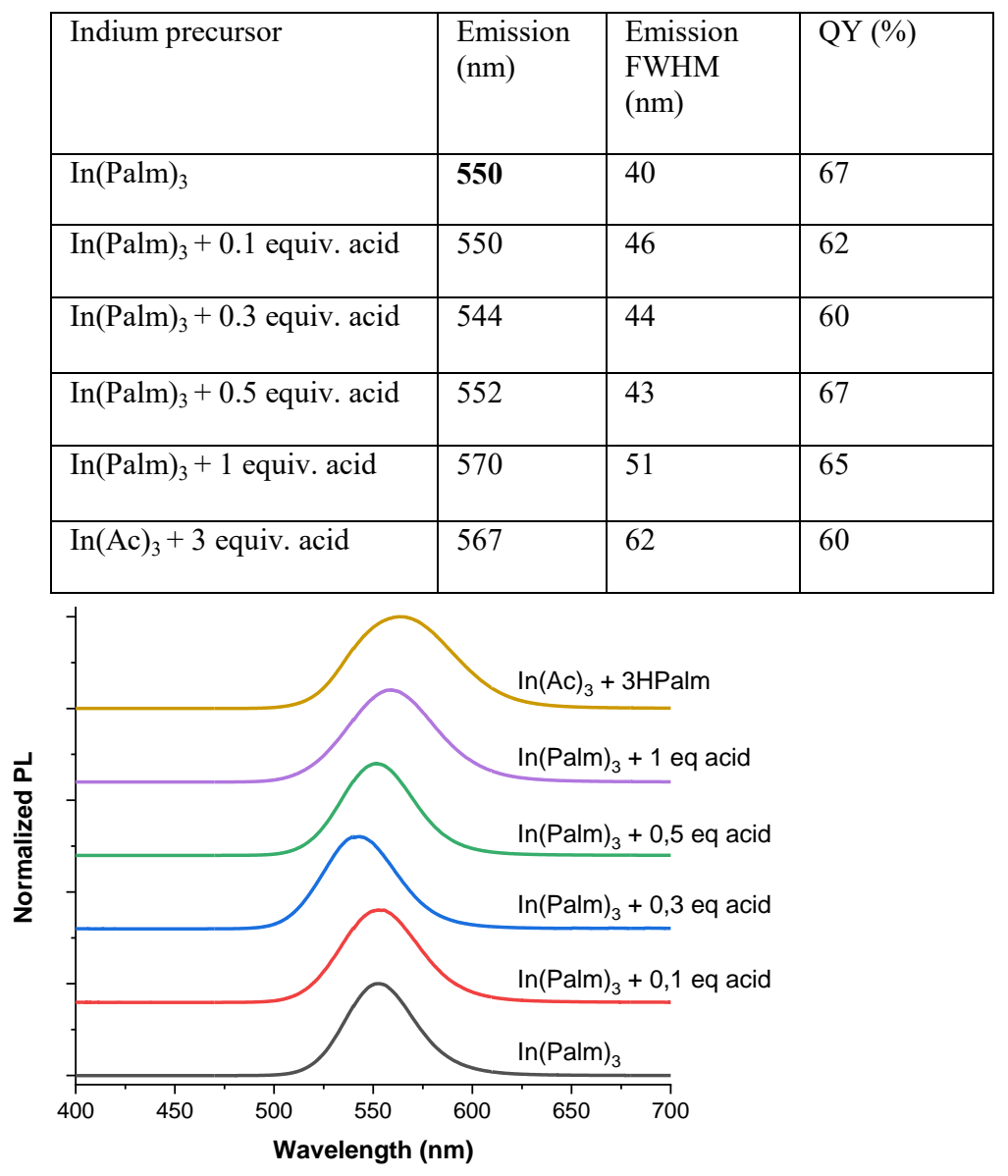

Figure 10. Photoluminescence spectra of core shell InP@ZnSeS QDs. 
The quantum yields of the final QDs were similar, therefore implying that the QY was reliant solely on the nature of the shell, and not the presence of acid during the synthesis of the core. Conversely, the FWHM clearly depended on the amount of acid used during synthesis, with the lowest FWHM obtained using an acid-free protocol. This demonstrated that preventing ripening was clearly important for the optical properties of the final material.

\section{CONCLUSION}

In summary, the purity of the indium precursor, i.e. acid free, was demonstrated to be key in obtaining high quality $\operatorname{InP}$ QDs from the reaction of $\mathrm{P}(\mathrm{TMS})_{3}$ and $\operatorname{In}(\mathrm{Palm})_{3}$. As shown by the subsequent NMR studies, it was linked to the inhibition of competitive pathways resulting from an uncontrolled partial hydrolysis of the $\mathrm{P}(\mathrm{TMS})_{3}$ precursor by carboxylic acids. Nucleation was shown to only have occurred above $210^{\circ} \mathrm{C}$ in the absence of acid, whereas substoichiometric amounts of acid were able to initiate nucleation at temperatures as low as $90^{\circ} \mathrm{C}$. Without the presence of acid, a slower growth occurred without a competitive ripening process. In this sense, acid free synthesis provided desirable growth conditions, as found in CdSe QD synthesis. Further studies are currently underway to lower FWHM and tune emission wavelength while maintaining a size focusing regime. This study was able to show that the presence of variable amounts of free acid as found in "classical" InP syntheses amounted for their poorer reproducibility in addition to their variable and worse optical properties. This work has hoped to contribute to the improvement of available methodologies for the synthesis of luminescent InP QDs. For instance, synthesizing larger InP nanocrystals in order to make red luminescent objects with sharp emission spectra remains a major challenge. The most efficient way to achieve this as 
described in the literature would be to slowly add, after initial QD formation, an $\operatorname{In}(\mathrm{RCOO})_{3^{-}}$ $\mathrm{P}(\mathrm{TMS})_{3}$ complex prepared at low temperatures. ${ }^{18,32}$ This process, however, often results in the broadening of the emission spectrum when compared with green QDs. This work suggests that these complexes might in fact be mixtures of different species due to the amount of free acid in solution. Using a clean indium carboxylate precursor to prepare these complexes could lead to a far greater control over growth and substantial increase in size focusing, thus, paving the way for the synthesis of greatly enhanced red-light emitting InP quantum dots.

\section{ASSOCIATED CONTENT}

Supporting Information contains experimental procedures, NMR, IR as well as absorption spectra of products and reactions; TEM pictures of InP/ZnSeS QD.

\section{AUTHOR INFORMATION}

\section{Corresponding Author}

*Nicolas Mézailles : mezailles@chimie.ups-tlse.fr

*Benoit Dubertret : benoit.dubertret@nexdot.fr

\section{Author Contributions}

The manuscript was written through contributions of all authors. All authors have given approval to the final version of the manuscript.

\section{Funding Sources}


This work was supported by the "Agence nationale de la recherche et des technologies" under convention CIFRE $N^{\circ}$ 2017/0502.

\section{ACKNOWLEDGMENT}

We would like to acknowledge Stéphane Massou for assistance with NMR. We would like to acknowledge Wilfried Solo Ojo for helpful discussion and critical reading of the manuscript. We would like to acknowledge Félix Boussoufi for assistance in collecting Powder X-ray diffraction patterns and Scott Willis for proof-reading of the manuscript. Solvay is acknowledged for a generous gift of phosphines.

\section{ABBREVIATIONS}

QDs, quantum dots; $\mathrm{P}(\mathrm{TMS})_{3}$, tris(trimethylsilyl)phosphine; ODE, 1-Octadecene; TPP, triphenylphosphine; MSC, magic-size clusters; TOP, trioctylphosphine; HWHM, half width at half maximum; FWHM, full width at half maximum; QY, quantum yield.

\section{REFERENCES}

(1) Murray, C. B.; Norris, D. J.; Bawendi, M. G. Synthesis and Characterization of Nearly Monodisperse CdE (E = Sulfur, Selenium, Tellurium) Semiconductor Nanocrystallites. J. Am. Chem. Soc. 1993, 115 (19), 8706-8715. https://doi.org/10.1021/ja00072a025.

(2) Reiss, P.; Carrière, M.; Lincheneau, C.; Vaure, L.; Tamang, S. Synthesis of Semiconductor Nanocrystals, Focusing on Nontoxic and Earth-Abundant Materials. Chem. Rev. 2016, 116 (18), 10731-10819. https://doi.org/10.1021/acs.chemrev.6b00116.

(3) Marşan, D.; Şengül, H.; Özdil, A. M. A. Comparative Assessment of the Phase Transfer Behaviour of $\mathrm{InP} / \mathrm{ZnS}$ and $\mathrm{CuInS} / \mathrm{ZnS}$ Quantum Dots and $\mathrm{CdSe} / \mathrm{ZnS}$ Quantum Dots under Varying Environmental Conditions. Environmental Science: Nano 2019, 6 (3), 879-891. https://doi.org/10.1039/C8EN01073K.

(4) Cui, J.; Beyler, A. P.; Marshall, L. F.; Chen, O.; Harris, D. K.; Wanger, D. D.; Brokmann, X.; Bawendi, M. G. Direct Probe of Spectral Inhomogeneity Reveals Synthetic Tunability of Single- 
Nanocrystal Spectral Linewidths. Nature Chemistry 2013, 5 (7), 602-606. https://doi.org/10.1038/nchem.1654.

(5) Janke, E. M.; Williams, N. E.; She, C.; Zherebetskyy, D.; Hudson, M. H.; Wang, L.; Gosztola, D. J.; Schaller, R. D.; Lee, B.; Sun, C.; Engel, G. S.; Talapin, D. V. Origin of Broad Emission Spectra in InP Quantum Dots: Contributions from Structural and Electronic Disorder. J. Am. Chem. Soc. 2018, 140 (46), 15791-15803. https://doi.org/10.1021/jacs.8b08753.

(6) Allen, P. M.; Walker, B. J.; Bawendi, M. G. Mechanistic Insights into the Formation of InP Quantum Dots. Angewandte Chemie International Edition 2010, 49 (4), 760-762. https://doi.org/10.1002/anie.200905632.

(7) Cossairt, B. M. Shining Light on Indium Phosphide Quantum Dots: Understanding the Interplay among Precursor Conversion, Nucleation, and Growth. Chem. Mater. 2016, 28 (20), 7181-7189. https://doi.org/10.1021/acs.chemmater.6b03408.

(8) Gary, D. C.; Glassy, B. A.; Cossairt, B. M. Investigation of Indium Phosphide Quantum Dot Nucleation and Growth Utilizing Triarylsilylphosphine Precursors. Chem. Mater. 2014, 26 (4), 1734-1744. https://doi.org/10.1021/cm500102q.

(9) Harris, D. K.; Bawendi, M. G. Improved Precursor Chemistry for the Synthesis of III-V Quantum Dots. J. Am. Chem. Soc. 2012, 134 (50), 20211-20213. https://doi.org/10.1021/ja309863n.

(10) Liu, Z.; Kumbhar, A.; Xu, D.; Zhang, J.; Sun, Z.; Fang, J. Coreduction Colloidal Synthesis of III-V Nanocrystals: The Case of InP. Angewandte Chemie International Edition 2008, 47 (19), 3540-3542. https://doi.org/10.1002/anie.200800281.

(11) An, S.; Cho, H.; Jeon, H. B.; Kim, S.-W. InPZnS Alloy Quantum Dots with Tris(Hexylthio)Phosphine as a Dual Anionic Precursor. Nanoscale 2018, 10 (6), 3014-3019. https://doi.org/10.1039/C7NR08211H.

(12) Mordvinova, N.; Vinokurov, A.; Dorofeev, S.; Kuznetsova, T.; Znamenkov, K. Phosphine Synthetic Route Features and Postsynthetic Treatment of InP Quantum Dots. Journal of Alloys and Compounds 2014, 582, 43-49. https://doi.org/10.1016/j.jallcom.2013.08.003.

(13) Li, L.; Protière, M.; Reiss, P. Economic Synthesis of High Quality InP Nanocrystals Using Calcium Phosphide as the Phosphorus Precursor. Chem. Mater. 2008, 20 (8), 2621-2623. https://doi.org/10.1021/cm7035579.

(14) Tessier, M. D.; Dupont, D.; De Nolf, K.; De Roo, J.; Hens, Z. Economic and Size-Tunable Synthesis of InP/ZnE (E = S, Se) Colloidal Quantum Dots. Chem. Mater. 2015, 27 (13), 48934898. https://doi.org/10.1021/acs.chemmater.5b02138. 
(15) Matsumoto, T.; Maenosono, S.; Yamaguchi, Y. Organometallic Synthesis of InP Quantum Dots Using Tris(Dimethylamino)Phosphine as a Phosphorus Source. Chem. Lett. 2004, 33 (11), 1492-1493. https://doi.org/10.1246/cl.2004.1492.

(16) Buffard, A.; Dreyfuss, S.; Nadal, B.; Heuclin, H.; Xu, X.; Patriarche, G.; Mézailles, N.; Dubertret, B. Mechanistic Insight and Optimization of InP Nanocrystals Synthesized with Aminophosphines. Chem. Mater. 2016, 28 (16), 5925-5934. https://doi.org/10.1021/acs.chemmater.6b02456.

(17) Xie, L.; Harris, D. K.; Bawendi, M. G.; Jensen, K. F. Effect of Trace Water on the Growth of Indium Phosphide Quantum Dots. Chem. Mater. 2015, 27 (14), 5058-5063. https://doi.org/10.1021/acs.chemmater.5b01626.

(18) Xu, Z.; Li, Y.; Li, J.; Pu, C.; Zhou, J.; Lv, L.; Peng, X. Formation of Size-Tunable and Nearly Monodisperse InP Nanocrystals: Chemical Reactions and Controlled Synthesis. Chem. Mater. 2019, 31 (14), 5331-5341. https://doi.org/10.1021/acs.chemmater.9b02292.

(19) Battaglia, D.; Peng, X. Formation of High Quality InP and InAs Nanocrystals in a Noncoordinating Solvent. Nano Lett. 2002, 2 (9), 1027-1030. https://doi.org/10.1021/n1025687v.

(20) Narayanaswamy, A.; Xu, H.; Pradhan, N.; Kim, M.; Peng, X. Formation of Nearly Monodisperse $\mathrm{In}_{2} \mathrm{O}_{3}$ Nanodots and Oriented-Attached Nanoflowers: Hydrolysis and Alcoholysis vs Pyrolysis. J. Am. Chem. Soc. 2006, 128 (31), 10310-10319. https://doi.org/10.1021/ja0627601.

(21) Gary, D. C.; Cossairt, B. M. Role of Acid in Precursor Conversion During InP Quantum Dot Synthesis. Chem. Mater. 2013, 25 (12), 2463-2469. https://doi.org/10.1021/cm401289j.

(22) Cros-Gagneux, A.; Delpech, F.; Nayral, C.; Cornejo, A.; Coppel, Y.; Chaudret, B. Surface Chemistry of InP Quantum Dots: A Comprehensive Study. J. Am. Chem. Soc. 2010, 132 (51), 18147-18157. https://doi.org/10.1021/ja104673y.

(23) Virieux, H.; Le Troedec, M.; Cros-Gagneux, A.; Ojo, W.-S.; Delpech, F.; Nayral, C.; Martinez, H.; Chaudret, B. InP/ZnS Nanocrystals: Coupling NMR and XPS for Fine Surface and Interface Description. J. Am. Chem. Soc. 2012, 134 (48), 19701-19708. https://doi.org/10.1021/ja307124m.

(24) Won, Y.-H.; Cho, O.; Kim, T.; Chung, D.-Y.; Kim, T.; Chung, H.; Jang, H.; Lee, J.; Kim, D.; Jang, E. Highly Efficient and Stable InP/ZnSe/ZnS Quantum Dot Light-Emitting Diodes. Nature 2019, 575 (7784), 634-638. https://doi.org/10.1038/s41586-019-1771-5. 
(25) Ramasamy, P.; Kim, N.; Kang, Y.-S.; Ramirez, O.; Lee, J.-S. Tunable, Bright, and NarrowBand Luminescence from Colloidal Indium Phosphide Quantum Dots. Chem. Mater. 2017, 29 (16), 6893-6899. https://doi.org/10.1021/acs.chemmater.7b02204.

(26) Gary, D. C.; Terban, M. W.; Billinge, S. J. L.; Cossairt, B. M. Two-Step Nucleation and Growth of InP Quantum Dots via Magic-Sized Cluster Intermediates. Chem. Mater. 2015, 27 (4), 1432-1441. https://doi.org/10.1021/acs.chemmater.5b00286.

(27) Franke, D.; Harris, D. K.; Xie, L.; Jensen, K. F.; Bawendi, M. G. The Unexpected Influence of Precursor Conversion Rate in the Synthesis of III-V Quantum Dots. Angew. Chem. Int. Ed. 2015, 54 (48), 14299-14303. https://doi.org/10.1002/anie.201505972

(28) Basel, S.; Bhardwaj, K.; Pradhan, S.; Pariyar, A.; Tamang, S. DBU-Catalyzed One-Pot Synthesis of Nearly Any Metal Salt of Fatty Acid (M-FA): A Library of Metal Precursors to Semiconductor Nanocrystal Synthesis. ACS Omega 2020, 5 (12), 6666-6675. https://doi.org/10.1021/acsomega.9b04448.

(29) Moreels, I.; Fritzinger, B.; Martins, J. C.; Hens, Z. Surface Chemistry of Colloidal PbSe Nanocrystals. J. Am. Chem. Soc. 2008, 130 (45), 15081-15086. https://doi.org/10.1021/ja803994m.

(30) Ramasamy, P.; Ko, K.-J.; Kang, J.-W.; Lee, J.-S. Two-Step "Seed-Mediated" Synthetic Approach to Colloidal Indium Phosphide Quantum Dots with High-Purity Photo- and Electroluminescence. Chem. Mater. 2018, 30 (11), 3643-3647. https://doi.org/10.1021/acs.chemmater.8b02049.

(31) Kim, Y.; Ham, S.; Jang, H.; Min, J. H.; Chung, H.; Lee, J.; Kim, D.; Jang, E. Bright and Uniform Green Light Emitting InP/ZnSe/ZnS Quantum Dots for Wide Color Gamut Displays. ACS Appl. Nano Mater. 2019, 2 (3), 1496-1504. https://doi.org/10.1021/acsanm.8b02063.

(32) Friedfeld, M. R.; Johnson, D. A.; Cossairt, B. M. Conversion of InP Clusters to Quantum Dots. Inorg. Chem. 2019, 58 (1), 803-810. https://doi.org/10.1021/acs.inorgchem.8b02945. 
For table of content only

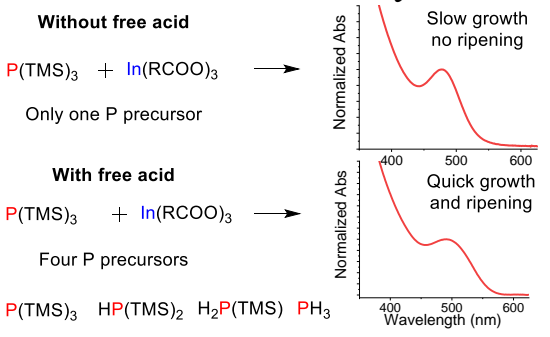

